\title{
Hydrophobic coiled-coil domains regulate the subcellular localization of human heat shock factor 2
}

\author{
Lynn A. Sheldon and Robert E. Kingston ${ }^{1}$ \\ Department of Molecular Biology, Massachusetts General Hospital, Boston, Massachusetts 02114 USA; Department of \\ Genetics, Harvard Medical School, Boston, Massachusetts 02115 USA
}

\begin{abstract}
HSF2, one of the heat shock transcription factors in mammalian cells, is localized to the cytoplasm during normal growth and moves to the nucleus upon activation. Heat shock transcription factors in metazoans contain four hydrophobic heptad repeat sequences, three in the amino terminus and one in the carboxyl terminus, which are predicted to form $\alpha$-helical coiled-coil structures analogous to the leucine zipper. Here, we show that point mutations in either of two amino-terminal zippers or in the carboxy-terminal zipper disrupt normal localization of HSF2 and cause it to be constitutively nuclear. We demonstrate further that two sequences immediately surrounding the amino-terminal zipper domain are required for nuclear localization. These sequences fit the consensus for a bipartite nuclear localization signal (NLS). We suggest that interactions between the amino- and carboxy-terminal zippers normally mask the NLS sequences of HSF2 and that these interactions are disrupted upon activation to expose the NLS sequences and allow transport of HSF2 to the nucleus. We conclude that zipper domains can regulate subcellular localization.
\end{abstract}

[Key Words: Heat shock factor 2; coiled-coil structures; leucine zipper; subcellular localization]

Received March 1, 1993; revised version accepted May 28, 1993.

Transcription factor function can be regulated by changes in DNA-binding ability, transcriptional activation ability, or subcellular compartmentalization. In contrast to the extensive characterization of DNA-binding domains and transcriptional activation domains, there are only a few examples of domains that are involved in regulating subcellular localization of transcription factors. Some transcription factors become constitutively nuclear after synthesis via an active process that requires specific basic amino acid motifs that function as nuclear localization signals (NLSs; for reviews, see Garcia-Bustos et al. 1991; Nigg et al. 1991; Silver 1991). Other factors are sequestered in the cytoplasm following synthesis and translocate to the nucleus only when induced by appropriate signals.

Factors can be sequestered in the cytoplasm either by protein-protein interactions or by post-translational modification. For example, interactions between Hsp90 and the glucocorticoid receptor (GR) have been proposed to mask the NLS sequences in the receptor, thus preventing transport of the GR to the nucleus. Upon activation by hormone, Hsp90 is released and GR is transported to the nucleus (Sanchez et al. 1985; Picard and Yamamoto 1987). A model for intramolecular masking was recently described by Henkel et al. (1992), in which

\footnotetext{
${ }^{1}$ Corresponding author.
}

the precursor for the p50 subunit of NF- $\mathrm{B}, \mathrm{p} 110$, folds back on itself in a manner that presumably masks the NLS sequences. Other subunits of NF-kB and other Relrelated factors are maintained in the cytoplasm by an interaction with proteins such as IкB via carboxy-terminal ankyrin repeats (Ghosh et al. 1990; Kieran et al. 1990; Davis et al. 1991; Haskill et al. 1991; for review, see Blank et al. 1992). In contrast to mechanisms that might sterically block the NLS, differential phosphorylation of residues near the NLS of SWI5 apparently alters the local charge and thus regulates the function of this NLS (Moll et al. 1991).

Heat shock transcription factor (HSF) has served as a model for characterizing regulatory mechanisms. HSF induces transcription of a set of genes following stress and in response to developmental signals (Craig 1980; Lindquist 1986; Morimoto et al. 1990). In metazoans and Schizosaccharomyces pombe, HSF is inactive as defined by its lack of binding to the heat shock element (HSE) during normal growth and is induced to bind to the HSE by heat shock. In budding yeasts HSF binds DNA constitutively and becomes a stronger transcriptional activator when the organism is stressed. HSF CDNAs have been isolated from a number of organisms, including humans, mouse, Drosophila, S. pombe, Saccharomyces cerevisiae, Kluyveromyces lactis, and tomatoes (Sorger and Pelham 1987; Weiderrecht et al. 1988; Clos et al. 1990; Scharf et al. 1990; Gallo et al. 1991; Jakobsen and 
Pelham 1991; Rabindran et al. 1991; Sarge et al. 1991; Schuetz et al. 1991). All HSFs contain a conserved DNAbinding domain at the amino terminus and an adjacent trimerization domain. The trimerization domain contains hydrophobic heptad repeats that have been shown to form $\alpha$-helical coiled-coil repeats similar to the leucine zipper (Landschulz et al. 1988; O'Shea et al. 1989; Peteranderl and Nelson 1992). Another conserved hydrophobic repeat that is predicted to form a zipper structure is found in the carboxy-terminal third of HSF (Clos et al. 1990; Sarge et al. 1991; Schuetz et al. 1991). It has recently been found that mutation of this zipper in either Drosophila or human HSF1 results in constitutive DNAbinding (Rabindran et al. 1993).

Two distinct cDNAs for HSF, HSF1, and HSF2 have been identified in mammalian cells (Rabindran et al. 1991; Sarge et al. 1991; Schuetz et al. 1991). It has been shown that HSF1 is strongly activated by heat to translocate to the nucleus and to bind to the HSE. HSF2 is activated to a significantly lesser degree by heat but can be activated in a hematopoietic cell line by hemin (Sistonen et al. 1992). This suggests that HSF1 and HSF2 may respond differentially to different stress signals. We demonstrate below that human HSF2 is cytoplasmic during normal growth conditions and moves to the nucleus in response to stress. We have identified two sequences that are necessary for nuclear transport of HSF2 following activation and have identified sequences that are required for cytoplasmic retention prior to activation. The data support a model in which interactions between the amino-terminal and carboxy-terminal zipper domains mask the NLS sequences when HSF2 is inactive. We propose that upon activation these interac- tions are disrupted to allow subsequent exposure of the NLS sequences and transport to the nucleus.

\section{Results}

Fractionation studies revealed that human HSF was found in cytoplasmic extracts at normal growth conditions and was in nuclear extracts after heat shock (Larson et al. 1988). These results raised the interesting possibility that the subcellular location of HSF could be altered by stress and, therefore, that subcellular compartmentalization could be a mode by which the activity of HSF is regulated.

We first determined the subcellular location of total HSF in HeLa cells growing at the normal temperature of $37^{\circ} \mathrm{C}$ or heat-shocked at $43^{\circ} \mathrm{C}$ by indirect immunofluorescence with a polyclonal antisera that recognizes both HSF1 and HSF2. At normal growth temperatures, fluorescent staining was observed in both the cell cytoplasm and the nucleus (Fig. 1). Following heat shock at $43^{\circ} \mathrm{C}$ for 30 min, cytoplasmic staining was significantly reduced and nuclear staining was increased with the appearance of a distinct punctate staining pattern (Fig. 1). This punctate pattern was apparent by $15 \mathrm{~min}$ (data not shown) and persisted for as long as $3 \mathrm{hr}$ following recovery from heat shock (Fig. 1). Intranuclear structures that exclude HSF are nucleoli (data not shown). These data demonstrate that total HSF is initially localized throughout the cell but concentrates in the nucleus following heat shock. Staining in Figure 1 and in all subsequent figures was analyzed using confocal imaging to distinguish cytoplasmic and nuclear compartments more effectively.

To distinguish introduced HSF2 from endogenous

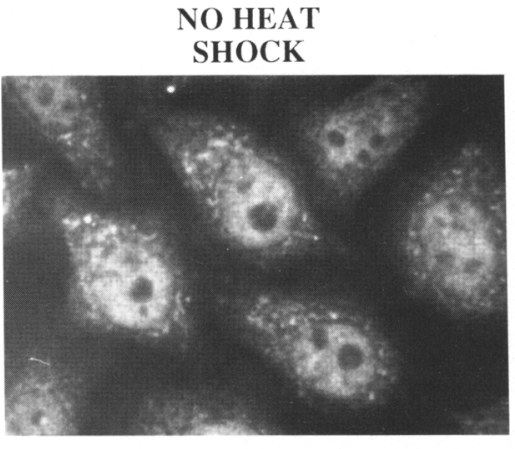

Figure 1. Time course of subcellular localization of endogenous human HSF in response to heat shock. HeLa cells were grown at $37^{\circ} \mathrm{C}$, heat-shocked for 30 or $60 \mathrm{~min}$ at $43^{\circ} \mathrm{C}$, or heatshocked for $60 \mathrm{~min}$ and allowed to recover for $3 \mathrm{hr}$. After treatment, cells were fixed, permeabilized, and incubated with a polyclonal antibody that recognizes endogenous HSFl and HSF2. Cells were visualized at $40 \times$ magnification by indirect immunofluorescence. $60^{\prime}$

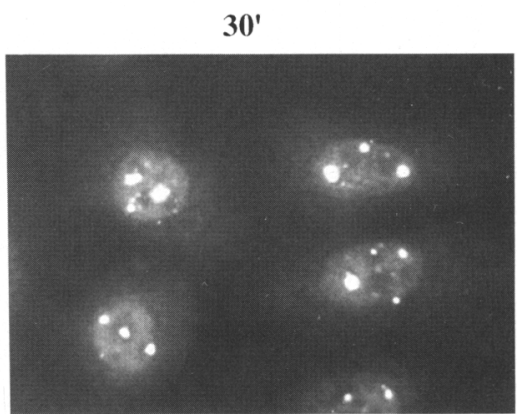

3 HR RECOVERY
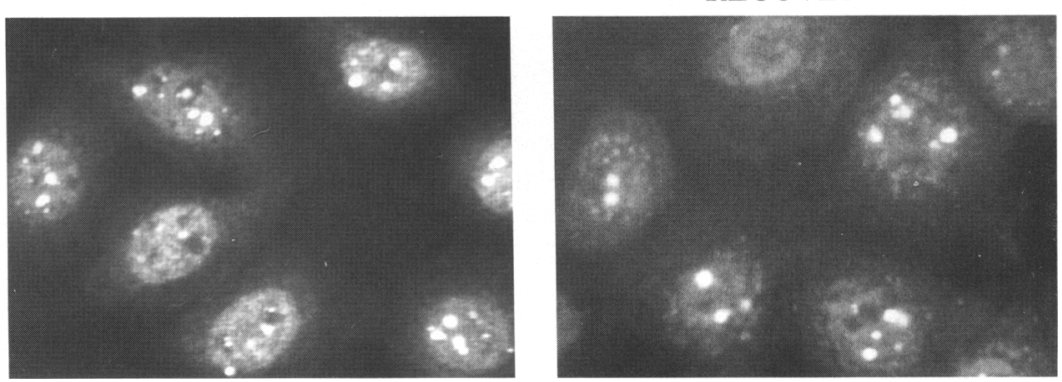
HSF1 and HSF2 in HeLa cells, a chimeric gene was made that encoded a fusion protein with 22 amino acids from the influenza hemagglutinin protein (Wilson et al. 1984) adjacent to the amino terminus of HSF2. This allowed visualization of the tagged HSF2 with a monoclonal antibody, mAb 12CA5, and a fluorescein-tagged second antibody. HSF2 was introduced into HeLa cells by transient transfection and was constitutively expressed from the cytomegalovirus (CMV) promoter. The epitope-tagged wild-type HSF2 was localized to the cytoplasm at the normal growth temperature of $37^{\circ} \mathrm{C}$ (Fig. 2). When cells were heat-shocked at $43^{\circ} \mathrm{C}$ for varying periods of time, some of the tagged HSF2 moved to the nucleus and formed punctate granules and some became perinuclear (Fig. 2). We conclude that HSF2 is translocated to the nucleus in response to heat shock but that this translocation is incomplete. The lack of complete nuclear localization following heat shock is consistent with previous data suggesting that heat shock primarily activates HSF1 and only weakly activates HSF2 (Sarge et al. 1993). Our observation that heat shock consistently caused transfected HSF2 to move to the nucleus provided a means of analyzing the sequences that are necessary for nuclear translocation. The cells shown in Figure 2 and all subsequent figures are representative of $>90 \%$ of the transfected cells observed in multiple transfection experiments.

\section{Sequences necessary for nuclear localization}

To understand how HSF2 is normally sequestered in the cytoplasm, we first needed to identify the NLSs. HSF2
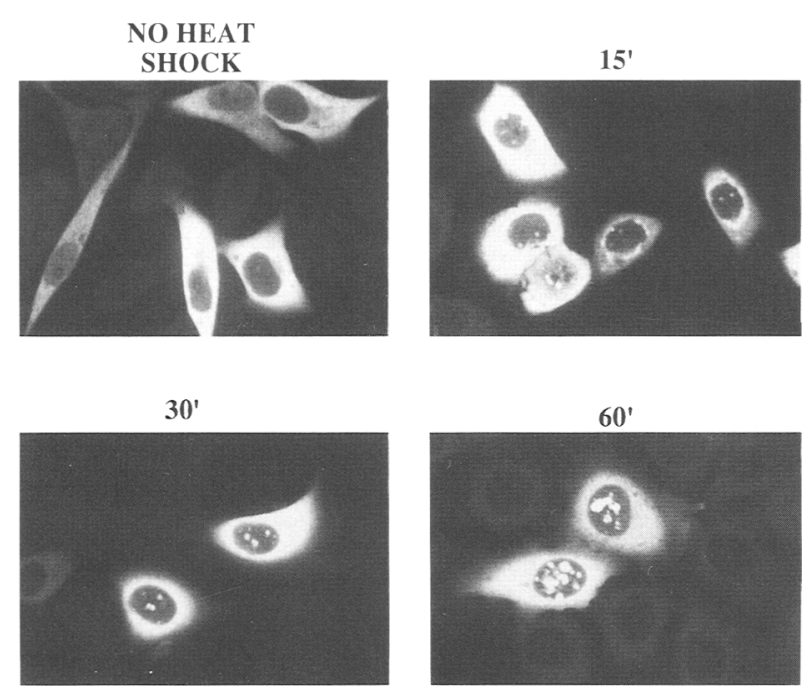

Figure 2. Time course of subcellular localization of HSF2 in HeLa cells in response to heat shock. HeLa cells were transfected with a construction expressing full-length HSF2 fused to the influenza hemagglutinin epitope tag at the amino terminus of HSF2. After $48 \mathrm{hr}$ at $37^{\circ} \mathrm{C}$, the cells were heat-shocked for 15 , 30 or $60 \mathrm{~min}$ at $43^{\circ} \mathrm{C}$. Following fixation and permeabilization, cells were incubated with mAb 12CA5, which recognizes the epitope on the tagged HSF2.

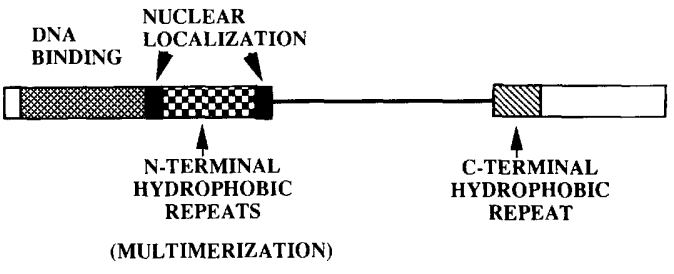

Figure 3. Schematic representation of HSF2 protein domains. The crosshatched area represents the DNA-binding domain; the solid boxes represent the sequences that fit the NLS sequence consensus; the checkered box designates the $5^{\prime}$ hydrophobic heptad repeat/multimerization domain; and the hatched box represents the 3 ' hydrophobic heptad repeat.

has an apparent molecular mass of $77-79 \mathrm{kD}$ (Sarge et al. 1993) and is therefore too big to freely diffuse into the nucleus. Inspection of the amino acid sequences of HSFl and HSF2 revealed two sequences that have the bipartite organization of the NLS found in nucleoplasmin and other nuclear proteins (Robbins et al. 1991). The bipartite NLS differs from the NLS of SV40 large T antigen (Kalderon et al. 1984) in that it has two basic domains that are functionally interdependent, separated by an $\sim 10$-amino-acid spacer region (Robbins et al. 1991). The most amino-terminal sequence in HSF2 that fits this consensus (termed NLS1) is located between Lys-108 and Arg-122, and lies between the DNA-binding domain and the proposed multimerization domain (see Fig. 3) (Schuetz et al. 1991). A second consensus sequence (termed NLS2) is located directly adjacent to the carboxy-terminal end of the multimerization domain and is found between Lys-195 and Lys-210.

Amino acid substitutions were made separately in each putative NLS of epitope-tagged HSF2 (Fig. 4A). In NLS1, Arg-109 was changed to Gly. In NLS2, amino acids 196-198 were changed from RKR to ASS. The mutant cDNAs were introduced into HeLa cells, and the cells were either kept at $37^{\circ} \mathrm{C}$ or were heat-shocked at $43^{\circ} \mathrm{C}$ for $90 \mathrm{~min}$. The localization pattern of both HSF2 variants mutated in the putative NLS sequences was dramatically altered from that of wild-type HSF2 (cf. Figs. 4B and 2). No movement from the cytoplasm to the nucleus was observed with either NLS mutant after 90 min of heat shock (Fig. 4B). These data demonstrate that NLS1 and NLS2 are necessary for nuclear translocation in HSF2. Although we have not determined whether the sequences are sufficient for nuclear localization, we refer to these sequences below as NLSs for brevity. The regulatory model proposed below requires only that these sequences be necessary for translocation of HSF2.

\section{Mutational analysis of the multimerization domain of HSF2}

The two bipartite NLSs in HSF1 and HSF2 directly flank the domain that is believed to be important for homomultimerization of HSF following heat shock (Sorger and Nelson 1989|. This multimerization domain is conserved in all eukaryotic HSFs, and the conserved region 
A

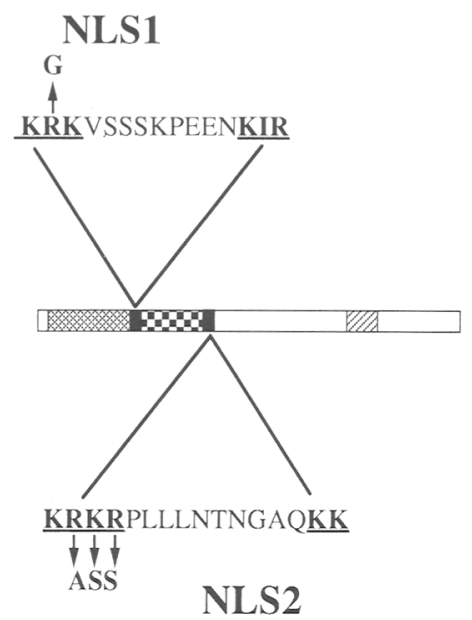

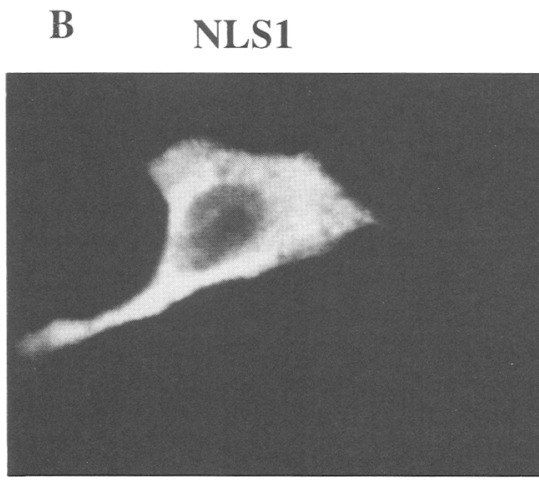

$43^{\circ} \mathrm{C}$

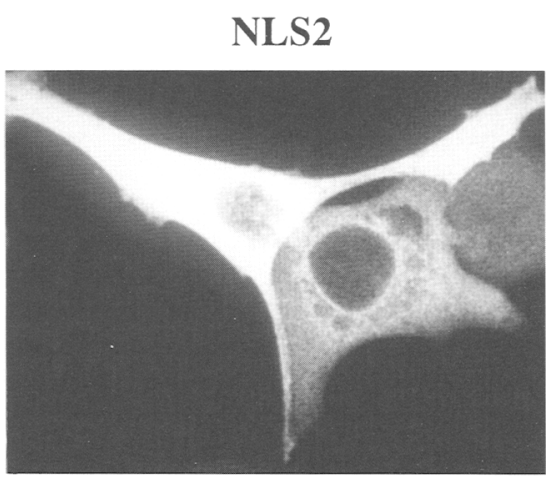

$43^{\circ} \mathrm{C}$

Figure 4. Mutations in NLS1 and NLS2. (A) Amino acid mutations were made in each of the two putative NLSs by site-directed mutagenesis to change Arg-109 to Gly in NLS1 and RKR $_{196-198}$ to ASS in NLS2. The template for the mutagenesis was full-length HSF2 that has the epitope tag fused to its amino terminus. Domain designations are as in Fig. 3. $(B)$ HeLa cells were transfected with constructions that had the mutations described in $A$. and following a heat shock at $43^{\circ} \mathrm{C}$ for 90 min the cells were fixed and permeabilized and the HSF2 was visualized by indirect immunofluorescent microscopy. The cells shown are representative of $>90 \%$ of the transfected cells observed in multiple transfection experiments.

in HSF2 extends from Glu-123 to Leu-194. It contains three hydrophobic heptad repeats, referred to as HR1, HR2, and HR3 (see Fig. 6A, below), which are predicted to form $\alpha$-coiled-coil repeats analagous to the leucine zipper (Landschulz et al. 1988; O'Shea et al. 1989; Peteranderl and Nelson 1992). Because this region is very hydrophobic we thought that it might be involved in masking the NLSs prior to heat shock either by interaction with another protein or by adopting a conformation that blocks NLS function.

We first deleted the entire multimerization domain from Glu-124 to Val-192, leaving 4 amino acids between the two intact NLSs (Fig. 5A). HSF2 deleted for these sequences was nuclear at $37^{\circ} \mathrm{C}$ (Fig. $5 \mathrm{~B}$ ). Interestingly, no punctate nuclear staining was seen even after a $43^{\circ} \mathrm{C}$ heat shock in contrast to the punctate staining pattern observed with the full-length protein at $43^{\circ} \mathrm{C}$ (cf. Figs. 5B and 21 .

To determine whether sequestration in the cytoplasm required a specific hydrophobic repeat, we analyzed the localization of HSF2 with amino acid mutations in HR1 and HR2 (Fig. 6A). The mutations in HR1 consisted of changes in Leu-157 $\rightarrow$ Val and Leu-164 $\rightarrow$ Val, in addition to an amino acid change just outside of the repeat of Arg-165 $\rightarrow$ Leu. The amino acid changes in HRl resulted in a constitutively nuclear phenotype following expres-
B

A

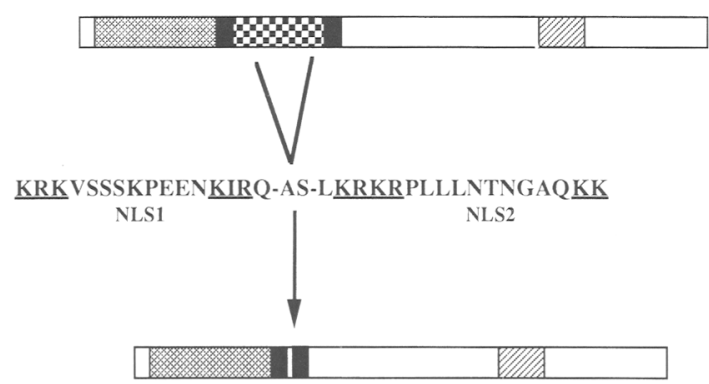

$37^{\circ} \mathrm{C}$

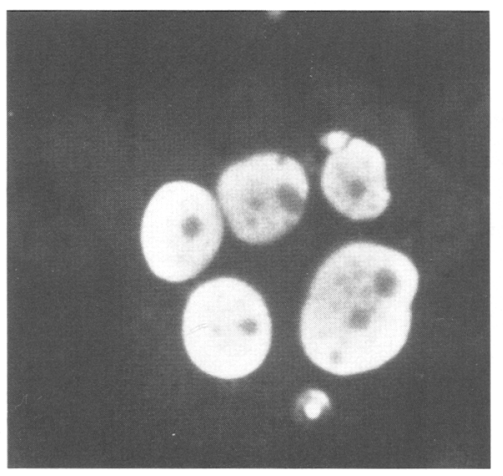

$43^{\circ} \mathrm{C}$

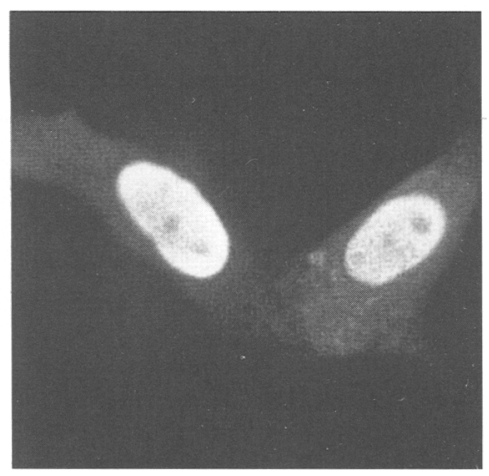

Figure 5. Deletion mutagenesis of the amino-terminal hydrophobic repeat/multimerization domain. (A) Schematic representation of the amino-terminal hydrophobic repeat domain deleted by site-directed mutagenesis that leaves the two NLSs intact. The two clusters of basic amino acids comprising the basic bipartite portion of the NLSs are underlined. Domain designations are as in Fig. 3. (B) HeLa cells were transfected with the construction containing the deletion mutation and maintained at $37^{\circ} \mathrm{C}$ or heat-shocked at $43^{\circ} \mathrm{C}$ for 90 min. HSF2 was visualized with mAb $12 \mathrm{CA} 5$ by indirect immunofluorescence. The $37^{\circ} \mathrm{C}$ cell is multinucleate. 


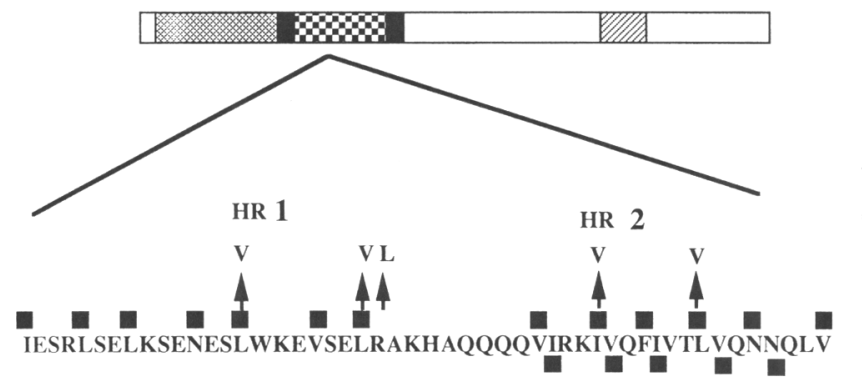

HR 3

2 OVERLAPPING HYDROPHOBIC REPEATS
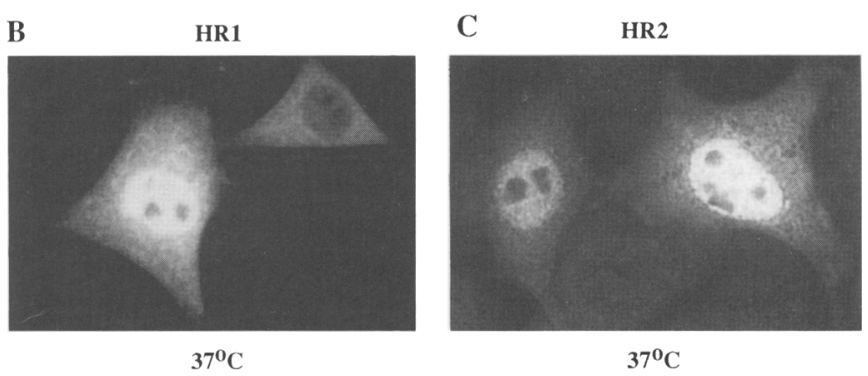

Figure 6. Point mutations in the amino-terminal hydrophobic heptad repeats. $(A)$ Amino acid mutations were made in hydrophobic repeat 1 (HR1) and hydrophobic repeat 2 (HR2) by site-directed mutagenesis. Amino acid changes are indicated by arrows; and amino acids predicted to comprise a $4 / 3$ coiled-coil motif are indicated by solid boxes. (B) A HeLa cell that was transfected with the mutated HR1 maintained at $37^{\circ} \mathrm{C}$. HSF2 was visualized by indirect immunofluorescence with mAb 12CA5. The cell in the upper right-hand corner of the field is untransfected and represents background staining. C. HeLa cells that were transfected with the HR2 mutation and maintained at $37^{\circ} \mathrm{C}$, visualized as in $B$.

sion in HeLa cells (Fig. 6B). Mutations made in HR2 changed Ile-178 $\rightarrow$ Val and Leu- $185 \rightarrow$ Val. The mutated construction $\mathrm{HR} 2$ was also nuclear at $37^{\circ} \mathrm{C}$ when expressed in HeLa cells (Fig. 6C). Neither mutation in HR1 or HR2 displayed punctate staining even at heat shock temperatures (data not shown). These results imply that two of the three amino-terminal hydrophobic repeats play a role in maintaining HSF2 in the cytoplasm at nonheat shock temperatures, perhaps through interactions that mask the NLSs. These experiments also demonstrate that mutations altering the trimerization domain eliminate the punctate staining pattern that is observed following heat shock (cf. Fig. 5B with Figs. 2 and 7C; data not shown).

\section{A carboxy-terminal protein domain of HSF2 that has a role in cytoplasmic retention}

The carboxy-terminal hydrophobic repeat of HSF2 is conserved in all mammalian HSFs, in Drosophila HSF, and in S. pombe and K. lactis HSFs (Clos et al. 1990; Gallo et al. 1991; Jakobsen and Pelham 1991; Rabindran et al. 1991; Sarge et al. 1991; Schuetz et al. 1991) and has the characteristic $4 / 3$ hydrophobic repeat of a zipper domain (Landschulz et al. 1988; O'Shea et al. 1989). It is not found in the budding yeast $S$. cerevisiae. This structural difference in HSFs is interesting in terms of possible regulation because HSF in S. cerevisiae is constitutively bound to DNA, whereas HSF in mammals, Drosophila, and $S$. pombe is not bound to DNA until it is activated by stress. The carboxy-terminal hydrophobic repeat has been implicated recently in regulating the inducibility of DNA binding of human HSF1 and Drosophila HSF (Rabindran et al. 1993). In HSF2, it lies between Leu-360 and Leu-378.

To investigate whether the hydrophobic repeat in
HSF2 has a role in the regulation of subcellular compartmentalization, we analyzed a series of carboxy-terminal deletions (Fig. 7A). Truncation at Gln-444 (86 amino acids carboxy-terminal of the hydrophobic repeat) results in a protein that is regulated in the same manner as the full-length protein (i.e., cytoplasmic at $37^{\circ} \mathrm{C}$ and nuclear at $43^{\circ} \mathrm{C}$; data not shown). Deletion of the carboxy-terminal portion of the protein starting at Thr-395, which is 16 amino acids carboxy-terminal to the hydrophobic repeat, yields a protein that is mostly nuclear at $37^{\circ} \mathrm{C}$ and completely nuclear at $43^{\circ} \mathrm{C}$ (data not shown). Digestion of HSF2 at a site that truncates the protein in the middle of the hydrophobic repeat at Cys-369 results in the production of a protein that is nuclear at $37^{\circ} \mathrm{C}$ and $43^{\circ} \mathrm{C}$ (data not shown). These data led to the hypothesis that the hydrophobic repeat may have a role in the maintenance of HSF2 in the cytoplasm prior to activation by stress.

An amino acid mutation was made in the carboxyterminal zipper domain of HSF2 that changed Leu-371 to an alanine (Fig. 7B). When this mutant construction was expressed in HeLa cells, the mutant protein was constitutively nuclear (Fig. 7C). This confirmed the idea that the integrity of the carboxy-terminal heptad repeat is important for maintaining HSF2 in the cytoplasm prior to activation by stress. The mutant protein also displayed a dramatically punctate staining pattern even at $37^{\circ} \mathrm{C}$, in contrast to the pattern seen in mutations in the amino-terminal hydrophobic repeats (cf. Fig. $7 \mathrm{C}$ with Figs. $6 \mathrm{~B}$ and $6 \mathrm{C}$ ).

\section{Discussion}

Coiled-coil interactions involving zipper domains are necessary for the dimerization and subsequent DNA binding of numerous transcription factors and can regulate the specificity of dimerization of factors with the 
A
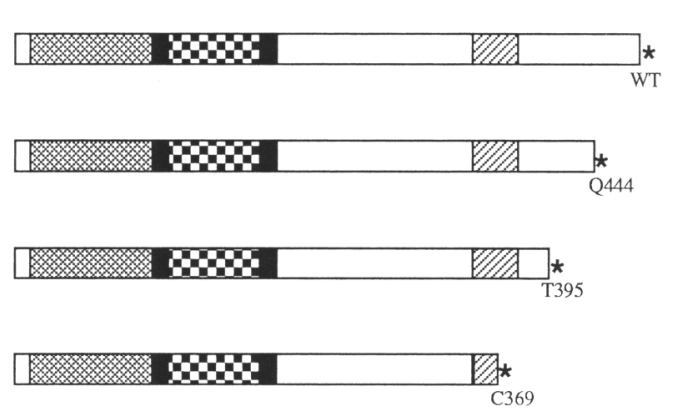

B

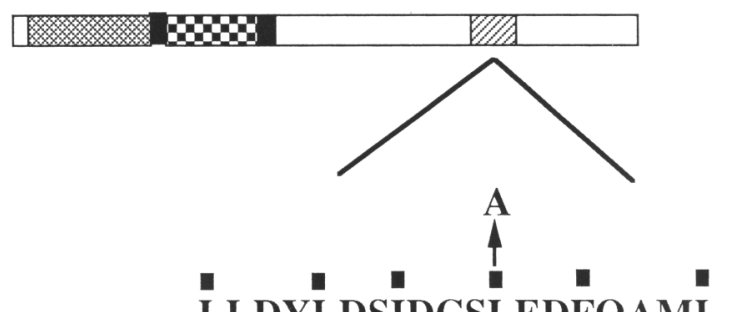

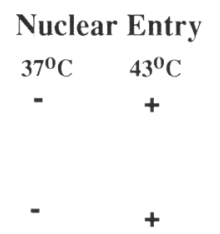

C

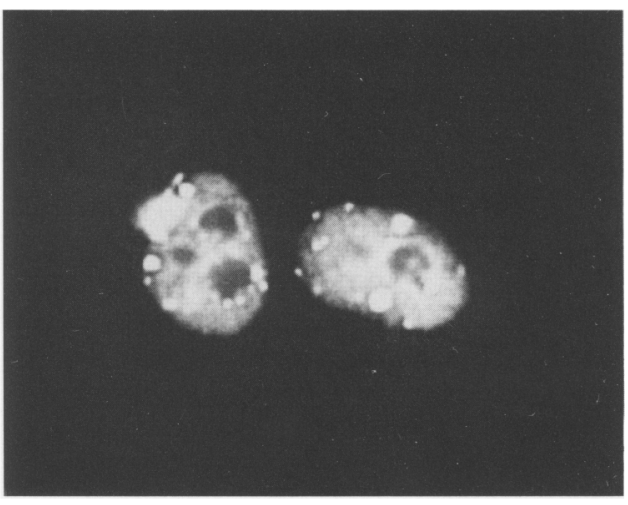

$37^{\circ} \mathrm{C}$

Figure 7. Deletion mutagenesis of the carboxyl terminus of HSF2. $(A \mid$ Deletions were made at the carboxyl terminus of the HSF2 construction fused to the epitope tag. The last amino acid in the protein is indicated, followed by a stop codon $\left({ }^{\star}\right)$. HeLa cells were transfected with one of the three deletions or the full-length (WT) HSF2, and the subcellular location of the deleted protein was visualized by indirect immunofluorescence with mAb 12CA5. The cells were either maintained at $37^{\circ} \mathrm{C}$ or heat-shocked for 90 min at $43^{\circ} \mathrm{C}$. (B) A single amino acid mutation was made in the carboxy-terminal hydrophobic repeat of HSF2. The Leu-371 $\rightarrow$ Ala change made by site-directed mutagenesis is indicated by an arrow. The amino acids indicated by solid boxes comprise the predicted $4 / 3$ coiled-coil motif. $(C)$ HeLa cells transfected with the point mutation Leu-371 $\rightarrow$ Ala was visualized by indirect immunofluorescence with mAb 12CA5. Cells were maintained at $37^{\circ} \mathrm{C}$.

basic leucine zipper (bZIP) domain (Landschulz et al. 1988; Agre et al. 1989; Hai et al. 1989; Kouzarides and Ziff 1989; O'Shea et al. 1989; Ivashkiv et al. 1990; Roman et al. 1990; Cao et al. 1991; Hai and Curran 1991; Williams et al. 1991; Schindler et al. 1992). The data presented above imply that coiled-coil interactions can also play a central role in regulating subcellular localization. Sequestration of transcription factors in the cytoplasm, where they cannot stimulate gene expression, is an effective and commonly used regulatory mechanism. Factors such as NF-kB and GR are held in the cytoplasm by interactions that involve either ankyrin repeats (NF$\kappa B$ and IкB) (Ghosh et al. 1990; Kieran et al. 1990; for review, see Blank et al. 1992) or an unspecified type of interaction (GR and Hsp90) (Sanchez et al. 1985; Picard and Yamamoto 1987). We demonstrate above that HSF2 is normally sequestered in the cytoplasm and that three separate zipper domains, two in the amino terminus and one in the carboxyl terminus of the protein, are required to maintain this cytoplasmic localization.

The hydrophobic heptad repeat sequences in HSF2 could function to regulate localization by intramolecular interactions, by interactions with other proteins, or by both types of interactions. An attractive model is that the amino- and carboxy-terminal hydrophobic repeats interact directly with each other to hold HSF2 in a con- formation that shields the sequences necessary for nuclear localization (NLS1 and NLS2) that lie immediately adjacent to the amino-terminal hydrophobic repeats (Fig. $8 \mathrm{~A}, \mathrm{~B})$. Human HSF undergoes a transition from a monomer to a trimer upon activation, consistent with a model for intramolecular interactions prior to activation and intermolecular interactions between amino-terminal domains following activation. A similar model has recently been proposed for regulation of the DNA-binding activities of human HSFl and Drosophila HSF based on the observation that mutation of the carboxy-terminal hydrophobic repeats of these factors causes constitutive binding to the HSE (Rabindran et al. 1993). Amino acid mutations in the carboxy-terminal hydrophobic repeat therefore result in constitutive DNA binding of HSFl (Rabindran et al. 1993) and constitutive nuclear localization of HSF2 (Fig. 7), arguing that the same interactions might regulate both properties of HSF. We have attempted to analyze the effects of our point mutations on regulated DNA binding by HSF2 but have been unable to detect binding by either wild-type or mutant HSF2 following transfection of mammalian cells (data not shown).

Here, we demonstrate that mutations in the aminoterminal hydrophobic repeats also result in constitutive nuclear localization, consistent with the hypothesis of 
$\mathbf{A}$

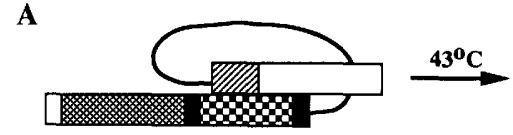

OR

B

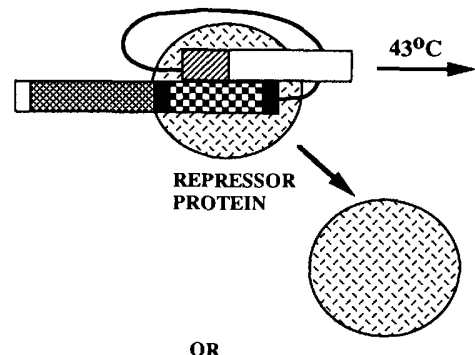

OR

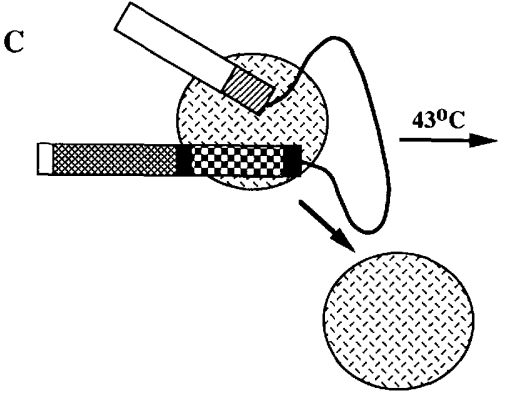

Figure 8. Models for HSF regulation. (A) In the first model, HSF2 is in a folded conformation at $37^{\circ} \mathrm{C}$, which allows the amino-terminal and carboxy-terminal hydrophobic repeats to interact with and stabilize the inactive monomeric conformation. An interaction of this type might result in the maintenance of the cytoplasmic location of HSF2 by the masking of the NLSs. Upon induction, the interaction may be disrupted exposing the NLSs and the multimerization domain to allow transport to the nucleus and multimerization. $(B)$ As above, except an auxiliary protein may also interact with HSF2 to stabilize the inactive conformation. $(C)$ The third possible model involves stabilization of the inactive conformation by another protein that interacts with both hydrophobic repeats, but the repeats themselves do not interact with one another.

intramolecular interactions between the amino- and carboxy-terminal hydrophobic repeats. The amino-terminal hydrophobic repeats are required for trimerization and DNA binding by HSF and thus appear to play a dual role: Under normal growth conditions they function to sequester HSF2 in the cytoplasm, whereas following activation they multimerize to form a trimer that binds to DNA (Fig. 8). It is possible that the interacton of this amino-terminal domain with its partner is weakened by heat or other signals to release the domain and allow subsequent translocation and trimerization of the factor. Calcium, which can play a role in destabilizing proteinprotein interactions, is required for activation of HSF (Mosser et al. 1990; Price and Calderwood 1991). Perhaps there is a conformational change of HSF upon activation that is regulated by a destabilization of interactions between the amino-terminal hydrophobic repeat sequences and partners, and calcium facilitates this destabilization. Biophysical studies of human HSF demonstrate a change in frictional coefficient, implying a change in conformation, upon activation of HSF (J. Larson and R. Kingston, in prep.). The factor changes from an elongated, monomeric structure to an even more dramatically elongated, trimeric structure upon heat shock, as would be expected if the inactive factor is held in a more compact configuration that becomes extended upon activation (Fig. 8).

While the simplest hypothesis is that the carboxy- and amino-terminal coiled-coil domains interact with each other, these domains might also interact with other proteins. An observation that is not easily explained solely by intramolecular interactions is that mutations in both amino-terminal hydrophobic repeat sequences disrupt subcellular localization (Fig. 6). The carboxy-terminal hydrophobic repeat is 19 amino acids and the two aminoterminal hydrophobic sequences are 22 and 19 amino acids in length (see Figs. 6 and 7). It therefore seems unlikely that the carboxy-terminal hydrophobic repeat could interact with both amino-terminal sequences at the same time, although it is possible that the two amino-terminal zippers and the carboxy-terminal zipper might form a structure containing all three zippers as a trimerized coiled-coil. Alternatively, other proteins might interact with one of the amino-terminal hydrophobic repeats to stabilize intramolecular interactions between the amino- and carboxy-terminal repeats (Fig. 8B). Finally, there may not be an intramolecular interaction. In this latter case, the data imply that other factors would have to interact with three sets of hydrophobic repeats (two amino-terminal and one carboxy-terminal), with no single interaction being sufficient to maintain cytoplasmic localization (Fig. 8C).

This issue will not be resolved without an understanding of how tightly the amino- and carboxy-terminal repeats bind to each other. This need not be a strong interaction, as the presence of both repeats in the same molecule will dramatically increase the effective concentration of the repeats relative to each other. It is known that the activation of HSF is very sensitive to concentration; at low concentrations in vitro it is not possible to activate the DNA-binding ability of inactive HSF (J. Larson and R. Kingston, unpubl.). Perhaps this reflects a need for the amino-terminal repeats to be at concentrations high enough to outcompete an intramolecular interaction with the carboxy-terminal repeat.

Human HSF1 is homologous to HSF2 in both the amino- and carboxy-terminal hydrophobic repeats and appears by inspection to have similarly located NLSs yet is not found in the cytoplasm to the extent that HSF2 is (Sarge et al. 1993). This suggests that although coiledcoil interactions are required to block nuclear translocation of HSF2, they might not be sufficient. It is possible, if not likely, that some other structure unique to HSF2 also is critical for cytoplasmic localization. Deletion analysis has suggested that a short carboxy-terminal region of HSF1 might play a role in regulating DNA binding in addition to the zippers. The deletion to Q444 (Fig. 
7), which removes all but 3 amino acids of the homologous sequence in HSF2, is regulated in the same manner as the wild-type protein, arguing that this region is not involved in regulation of subcellular compartmentalization.

HSF forms punctate granules in the nucleus following heat shock. These granules are also seen at normal temperatures when a single amino acid change is made in the carboxy-terminal repeat of HSF2 (Fig. 7) but are not seen with any of the mutations in the amino-terminal repeats (Fig. 5; data not shown). Thus, the formation of these granules requires that the amino-terminal oligomerization domain be intact. This domain might either form an interaction with other factors that causes the punctate staining or might be required for an activity of HSF (i.e., DNA binding) that is necessary to localize HSF in these granules. These granules are not preferentially associated with the nucleolus (data not shown) or with any other obvious nuclear structure. The possible function of this subcompartmentalization of HSF in the nucleus is not understood.

\section{Materials and methods}

\section{DNA constructions}

HSF2 was excised from pHSF2-19 (Schuetz et al. 1991) at the HincII site, $5^{\prime}$ of the AUG start codon, filled in with the Klenow fragment of DNA polymerase, and digested with KpnI, which is found at the $3^{\prime}$ end of the gene in the pBluescript SK vector (Stratagene). The $\sim 2.5-\mathrm{kb}$ fragment was gel purified and ligated into the pCGN vector (Tanaka and Herr 1990). This puts the influenza hemagglutinin epitope tag (Field et al. 1988) at the $5^{\prime}$ end of HSF2, and its expression is driven by the CMV promoter that is constitutively expressed in HeLa cells.

Deletions were made at the carboxy terminus of pCGN/HSF2 by cutting at a PstI site, which truncates the protein at Cys-369, a SpeI site, which truncates the protein at Thr-395, or at the $A c c I$ site which truncates at Gln-444. The AccI and SpeI sites were filled in using the large fragment of DNA polymerase, and the PstI site was made blunt with T4 DNA polymerase. NheI linkers with stop codons in all three reading frames were ligated at each of the blunt restriction sites.

\section{Mutagenesis}

Site-directed mutagenesis of the plasmid pCGN/HSF2 was done by the method of Kunkel et al. (1987). Single-stranded, uracil-substituted DNA was prepared by transforming the dut ${ }^{-}$ung $^{-}$Escherichia coli strain R382 with pCGN/HSF2 that was then infected with M13R408 helper phage (Stratagene). Approximately 20 pmoles of a phosphorylated oligomer containing the mutation of interest was annealed to $\sim 1 \mu \mathrm{g}$ of singlestranded DNA in $20 \mathrm{~mm}$ Tris (pH 7.4) $2 \mathrm{~mm} \mathrm{MgCl}_{2}$, and $50 \mathrm{~mm}$ $\mathrm{NaCl}$. After annealing, $10 \times$ polymerization buffer $(100 \mathrm{~mm}$ Tris- $\mathrm{HCl}$ at $\mathrm{pH} 7.4,50 \mathrm{~mm} \mathrm{MgCl}_{2}, 10 \mathrm{~mm}$ ATP, $5 \mathrm{~mm}$ each dNTP, $20 \mathrm{mM}$ DTT) was added to the reaction, as well as 10 units of T4 DNA ligase and 1 unit of T4 DNA polymerase. The polymerization reaction was incubated for $15 \mathrm{~min}$ on ice followed by incubation overnight at $16^{\circ} \mathrm{C}$. The reaction was diluted to $80 \mu \mathrm{l}$ with TE ( $10 \mathrm{~mm}$ Tris at $\mathrm{pH} 8.0,2 \mathrm{mM}$ EDTA), and $5 \mu$ of that was used to transform E. coli strain JM109 by electroporation (Ausubel et al. 1992).

Following identification of clones containing mutations, the mutated regions were subcloned into plasmid pCGN/HSF2 to replace the homologous unmutated portion of HSF2 with the mutated portion. The entire mutated, subcloned portion of HSF2 was then sequenced (Sequenase, U.S. Biochemical) to confirm the mutation and ensure that the rest of the plasmid was not carrying an undesired mutation.

\section{Oligonucleotides synthesized for mutagenesis}

Boldface type indicates mutagenized bases. NLS1, GAG-AACATT-ACA-AGC-AAC-GTT-TCA-TCT; NLS2, GTG-AGT-TTA-AAA-GCT-AGC-CCT-CTA-CTT; $\Delta 5^{\prime} \mathrm{LZ}$, AAA-ATT-CGTCAG-GCT-AGC-TTA-AAA-CGT-AAA; 5'LZ-2V, GAG-AATGAG-TCC-GTT-TGG-AAG-GAG-GTG-TCA-GAA-GTA-CTA-GCA-AAG-C; 5'LZ(2), AAC-AGC-AAC-AAG-TTG-TTC-GAA-AGA-TTG-TCC-AGT-TTG-TTG-TTA-CAT-TGG-TT; 5'LZ(3), AAG-TTA-TTC-GAA-AGG-TTG-TCC-AGT-TTA-TTGTTA-CAG-TGG-TTC-AAA-ATA-AC; and 3'LZ-1A, GTA-TTGACT-GCA-GTG-CAG-AGG-ACT-TCC-AGG-CCA-TGG-CATCAG-GAA-GAC-AAT.

\section{Cell culture}

HeLa cells were grown in Dulbecco's minimum essential medium with $10 \%$ calf serum at $37^{\circ} \mathrm{C}, 5 \% \mathrm{CO}_{2}$. Heat shock was at $43^{\circ} \mathrm{C}$ for $1 \mathrm{hr}$ or for $90 \mathrm{~min}$ in a $5 \% \mathrm{CO}_{2}$ incubator.

\section{Transfections}

Cells were grown on glass slides from Cel-line Associates, Inc., and transfected using calcium phosphate precipitates (Ausubel et al. 1992) of individual plasmids at $20-40 \mu \mathrm{g} / \mathrm{ml}$. Cells were incubated with the precipitates for $16 \mathrm{hr}$, rinsed with $1 \times$ PBS, and fed with complete medium. Cells recovered for $48-72 \mathrm{hr}$ before experiments were done.

\section{Immunocytochemistry}

All procedures were at room temperature. Cells were rinsed with $1 \times$ PBS and fixed for $30 \mathrm{~min}$ in $3.7 \%$ formaldehyde in PBS. Permeabilization was in $1 \%$ Triton X-100 in PBS for $5 \mathrm{~min}$ followed by a brief rinse in $1 \times$ PBS

Primary and secondary antibodies were diluted $1 / 200$ in $0.5 \%$ BSA in PBS. Secondary antibodies were fluorescein-conjugated, affinity-purified goat anti-mouse IgG or goat anti-rabbit IgG from Cappel. The cells were incubated with the primary antibody for $1 \mathrm{hr}$ and the secondary antibody for $30 \mathrm{~min}$.

Following incubation with the antibodies, the cells were rinsed with $1 \times$ PBS. A few drops of $2 \% n$-propyl gallate in $30 \%$ $0.1 \mathrm{M}$ Tris ( $\mathrm{pH} 9.5$ ) and $70 \%$ glycerol were put on the cells to retard fading.

Cells were viewed using a Zeiss Axiophot at $40 \times$. Bio-Rad/ MRC software was used with the confocal microscopy.

\section{Antibodies}

The polyclonal antibody used in this study was raised in mice against HSF purified from heat-shocked HeLa cell nuclear extracts as described by Schuetz et al. (1991). The polyclonal immune ascites fluid was diluted $1 / 200$ in $0.5 \%$ BSA in PBS for the detection of both HSF1 and HSF2 in HeLa cells. Secondary antibody was fluorescein-conjugated goat affinity-purified antibody to mouse IgG diluted 1/200 (Cappel, Organon Teknika Corp., West Chester PA) or Texas Red-conjugated goat antirabbit IgG at $10 \mu \mathrm{g} / \mathrm{ml}$ (Molecular Probes, Eugene, OR).

$\mathrm{mAb} 12 \mathrm{CA} 5$, a subclone of $\mathrm{H} 26 \mathrm{DO}$, recognizes residues 75- 
110 of HAl (Wilson et al. 1984; Field et al. 1988). These residues are the amino-terminal 22-amino-acid epitope tag on the plasmid pCGN (Tanaka and Herr 1990). The antibody was kindly provided by Dr. Ian Wilson (Scripps Clinic and Research Foundation, La Jolla, CA).

\section{Acknowledgments}

We thank Gary Ruvkun and Paul Matsudaira for the use of their microscopes and especially Mark Chafel and Ya-Huei Tu for their invaluable help with confocal microscopy. We thank Tom Schuetz for the polyclonal antisera directed against HSF, Brian Seed for help in preparation of mAb 12CA5, Ian Wilson for the cell line for 12CA5, and Masafumi Tanaka for the plasmid pCGN. Our thanks go to Marjorie Oettinger, Gary Ruvkun, Jeffrey Larson, Kirstie Saltsman, and Catherine Kara for critical reading of the manuscript. This work was supported by grants from the National Institutes of Health (GM43901) and from Hoechst AG.

The publication costs of this article were defrayed in part by payment of page charges. This article must therefore be hereby marked "advertisement" in accordance with 18 USC section 1734 solely to indicate this fact.

\section{References}

Agre, P., P. Johnson, and S. McKnight. 1989. Cognate DNA binding specificity retained after leucine zipper exchange between GCN4 and C/EBP. Science 246: 922-925.

Ausubel, F.M., R. Brent, R.E. Kingston, D.D. Moore, J.G. Seidman, J.A. Smith, and K. Struhl, eds. 1992. Current protocols inmolecular biology. Greene Publishing Associates/Wiley-Interscience, New York.

Blank, V., P. Kourilsky, and I. Alain. 1992. NF-кB and related proteins: Rel/dorsal homologies meet ankyrin-like repeats. Trends Biochem. Sci. 17: 135-140.

Cao, Z., R. Umek, and S. McKnight. 1991. Regulated expression of three C/EBP isofoems during adiposide conversion of 3T3-L1 cells. Genes \& Dev. 5: 1538-1552.

Clos, J., J.T. Westwood, P.B. Becker, S. Wilson, K. Lambert, and C. Wu. 1990. Molecular cloning and expression of a hexameric Drosophila heat shock factor subject to negative regulation. Cell 63: 1085-1097.

Craig, Elizabeth A. 1980. The heat shock response. Crit. Rev. Biochem. 18: 239-280.

Davis, N., S. Ghosh, D.L. Simmons, P. Tempst, H.-C. Liou, D. Baltimore, and H.R. Bose Jr. 1991. Rel-associated pp40: An inhibitor of the Rel family of transcription factors. Science 253: 1268-1271.

Field, J., J.-I. Nikawa, D. Broek, B. MacDonald, L. Rodgers, I.A. Wilson, R.A. Lerner, and M. Wigler. 1988. Purification of a RASresponsive adenylyl cyclase complex from Saccharomyces cerevisiae by use of an epitope addition method. Mol. Cell. Biol. 8(5): 2159-2165.

Gallo, G.J., T.J. Schuetz, and R.E. Kingston. 1991. Regulation of heat shock factor in Schizosaccharomyces pombe more closely resembles regulation in mammals than in Saccharomyces cerevisiae. Mol. Cell. Biol. 11: 281-288.

Garcia-Bustos, J., J. Heitman, and M.N. Hall. 1991. Nuclear protein localization. Biochim. Biophys. Acta 1071: 83-101.

Ghosh, S., A.M. Gifford, L.R. Riviere, P. Tempst, G.P. Nolan, and D. Baltimore. 1990. Cloning of the p50 DNA binding subunit of NF-кB: Homology to rel and dorsal. Cell 62: 1019-1029.

Hai, T. and T. Curran. 1991. Cross-family dimerization of transcription factors FOS/JUN and ATF/CREB alters DNAbinding specificity. Proc. Natl. Acad. Sci. 88: 3720-3724.

Hai, T., F. Liu, W. Coukos, and M. Green. 1989. Transcription factor cDNA clones: An extensive family of leucine zipper proteins able to selectively form DNA-binding heterodimers. Genes \& Dev. 3: 2083-2090.

Haskill, S., A.A. Beg, S.M. Tompkins, J.S. Morris, A.D. Yurochko, A. Sampson-Johannes, K. Mondal, P. Ralph, and A.S. Baldwin Jr. 1991. Characterization of an immediate early gene induced in adherent monocytes that encodes IкB-like activity. Cell 65: 1281-1289.

Henkel, T., U. Zabel, K. Van Zee, J.M. Muller, E. Fanning, and P.A. Baeuerle. 1992. Intramolecular masking of the nuclear localization signal and dimerization domain in the precursor for the p50 NF-kB subunit. Cell 68: 1121-1133.

Ivashkiv, L.B., H.-C. Liou, C.J. Kara, W.W. Lamph, I.M. Verma., and L.H. Glimcher. 1990. mXBP/CRE-BP2 and c-Jun form a complex which binds to the cyclic AMP, but not to the 12-O-tetradecanoylphorbol-13-acetate, response element. Mol. Cell. Biol. 10: 1609-1621.

Jakobsen, B.K. and H.R.B. Pelham. 1991. A conserved heptapeptide restrains the activity of the yeast heat shock transcription factor. $E M B O J$. 10(2): 369-375.

Kalderon, D., B.L. Roberts, W.D. Richardson, and A.E. Smith. 1984. A short amino acid sequence able to specify nuclear location. Cell 39: 499-509.

Kieran, M., V. Blank, F. Logeat, J. Vandekerckhove, F. Lottspeich, O. Le Bail, M. Urban, P. Kourilsky, P.A. Baeuerle, and A. Israel. 1990. The DNA binding subunit of NF-кB is identical to factor KBF1 and homologous to the rel oncogene product. Cell 62: $1007-1018$.

Kouzarides, T. and E. Ziff. 1989. Leucine zippers of Fos, Jun and GCN4 dictate dimerization specificity and thereby control DNA binding. Nature 340: 568-571.

Kunkel, T.A., J.D. Roberts, and R.A. Zakour. 1987. Rapid and efficient site-specific mutagenesis without phenotypic selection. Methods Enzymol. 154: 367-382.

Landschulz, W.H., P.F. Johnson, and S.L. McKnight. 1988. The leucine zipper: A hypothetical structure common to a new class of DNA binding proteins. Science 240: 1759-1764.

Larson, J.S., T.J. Schuetz, and R.E. Kingston. 1988. Activation in vitro of sequence specific DNA by a human regulatory factor. Nature 335: 372-375.

Lindquist, S. 1986. The heat shock response. Annu. Rev. Biochem. 55: 1151-1191.

Moll, T., G. Tebb, U. Surana, H. Robitsch, and K. Nasmyth. 1991. The role of phosphorylation and the CDC28 protein kinase in cell cycle-regulated nuclear import of the $\mathrm{S}$. cerevisiae transcription factor SWI5. Cell 66: 743-758.

Morimoto, R.I., A. Tissieres, and C. Georgopoulos, eds. 1990. Stress proteins in biology and medicine. Cold Spring Harbor Laboratory Press, Cold Spring Harbor, New York.

Mosser, D.D., P.T. Kotzbauer, K.D. Sarge, and R.I. Morimoto. 1990. In vitro activation of heat shock transcription factor DNA-binding by calcium and biochemical conditions that affect protein conformation. Proc. Natl. Acad. Sci. 87: 37483752 .

Nigg, E.A., P.A. Baeuerle, and R. Luhrmann. 1991. Nuclear import-export: In search of signals and mechanisms. Cell 66: 15-22.

O'Shea, E.K., R. Rutkowski, W.F. Stafford III, and P.S. Kim. 1989. Preferential heterodimer formation by isolated leucine zippers from Fos and Jun. Science 245: 646-648.

Peteranderl, R. and H.C.M. Nelson. 1992. Trimerization of the heat shock transcription factor by a triple-stranded a-helical coiled-coil. Biochemistry 31: 12272-12276. 
Picard, D. and K. Yamamoto. 1987. Two signals mediate hormone-dependent nuclear localization of the glucocorticoid receptor. EMBO /. 6: 3333-3340.

Price, B.D. and S.K. Calderwood. 1991. $\mathrm{Ca}^{2+}$ is essential for multistep activation of the heat shock factor in permeabilized cells. Mol. Cell. Biol. 11: 3365-3368.

Rabindran, S.K., G. Giorgi, J. Clos, and C. Wu. 1991. Molecular cloning and expression of a human heat shock factor. Proc. Natl. Acad. Sci. 88: 6906-6910.

Rabindran, S.K., R.I. Haroun, J. Clos, J. Wisniewski, and C. Wu. 1993. Regulation of heat shock factor trimer formation: Role of a conserved leucine zipper. Science 259: 230-234.

Robbins, J., S.M. Dilworth, R.A. Laskey, and C. Dingwall. 1991. Two interdependent basic domains in nucleoplasmin nuclear targeting sequence: Indentification of a class of bipartite nuclear targeting sequence. Cell 64: 615-623.

Roman, C., J. Platero, J. Shuman, and K. Calame. 1990. Ig/ EBP-1: A ubiquitously expressed immunoglobulin enhancer bindng protein that is similar to C/EBP and heterodimerizes with C/EBP. Genes \& Dev. 4: 1404-1415.

Sanchez, E.R., D.O. Toft, M.J. Schlesinger, and W.B. Pratt. 1985. Evidence that the 90-kDa phosphoprotein associated with untransformed L-cell glucocorticoid receptor is a murine heat shock protein. J. Biol. Chem. 260: 12398-12401.

Sarge, K.D., V. Zimarino, K. Holm, C. Wu, and R.I. Morimoto. 1991. Cloning and characterization of two mouse heat shock factors with distinct inducible and constitutive DNA-binding ability. Genes \& Dev. 5: 1902-1911.

Sarge, K.D., S.P. Murphy, and R.I. Morimoto. 1993. Activation of heat shock gene transcription by HSFl involves oligomerization, acquisition of DNA-binding activity, and nuclear localization and can occur in the absence of stress. Mol. Cell. Biol. 13: 1392-1407.

Scharf, K.-D., S. Rose, W. Zott, F. Schoff, and L. Nover. 1990. Three tomato genes code for heat stress transcription factors with a region of remarkable homology to the DNA-binding domain of the yeast HSF. EMBO J. 9 (13): 4495-4501.

Schindler, U., A.E. Menkens, H. Beckman, J.R. Ecker, and A.R. Cashmore. 1992. Heterodimerization between the lightregulated ubiquitously expressed Arabidopsis GBF bZIP proteins. EMBO I. 11: 1261-1273.

Schuetz, T.J., G.J. Gallo, L. Sheldon, P. Tempst, and R.E. Kingston. 1991. Isolation for a cDNA for HSF2: Evidence for two heat shock factor genes in humans. Proc. Natl. Acad. Sci. 88: 6910-6915.

Silver, P.A. 1991. How proteins enter the nucleus. Cell 64: 489-497.

Sistonen, L., K.D. Sarge, B. Phillips, K. Abravaya, and R.I. Morimoto. 1992. Activation of heat shock factor 2 during hemin-induced differentiation of human erythroleukemia cells. Mol. Cell. Biol. 12: 4104-4111.

Sorger, P.K. and H.C.M. Nelson. 1989. Trimerization of a yeast transcriptional activator via a coiled-coil motif. Cell 59: 807-813.

Sorger, P.K. and H.R.B. Pelham. 1987. Purification and characterization of a heat-shock element binding protein from yeast. EMBO I. 6: 3035-3041.

Tanaka, M. and W. Herr. 1990. Differential transcriptional activation by Oct-1 and Oct-2: Interdependent activation domains induce Oct-2 phosphorylation. Cell 60: 375-386.

Weiderrecht, G., D. Seto, and C.S. Parker. 1988. Isolation of the gene encoding the $S$. cerevisiae heat shock transcription factor. Cell 62: 793-805.

Williams, S., C. Cantwell, and P. Johnson. 1991. Family of C/EBP-related proteins capable of forming covalently linked leucine zipper dimers in vitro. Genes \& Dev. 5: 1553-1567.
Wilson, I.A., H.L. Niman, R.A. Houghten, A.R. Cherenson, M.L. Connolly, and R.A. Lerner. 1984. The structure of an antigenic determinant in a protein. Cell 37: 767-778. 


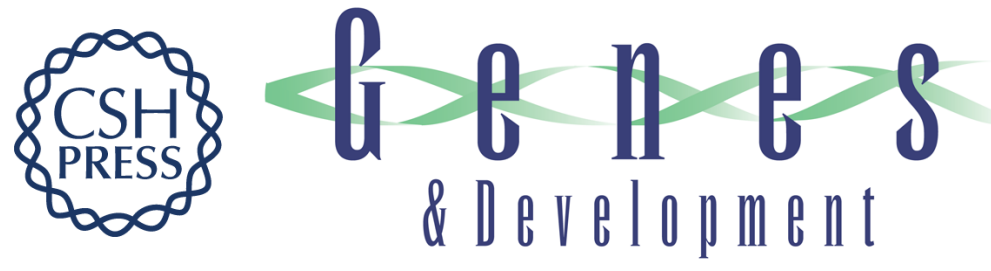

\section{Hydrophobic coiled-coil domains regulate the subcellular localization of human heat shock factor 2.}

$L A$ Sheldon and R E Kingston

Genes Dev. 1993, 7:

Access the most recent version at doi:10.1101/gad.7.8.1549

References This article cites 48 articles, 20 of which can be accessed free at:

http://genesdev.cshlp.org/content/7/8/1549.full.html\#ref-list-1

License

Email Alerting

Service

Receive free email alerts when new articles cite this article - sign up in the box at the top right corner of the article or click here.

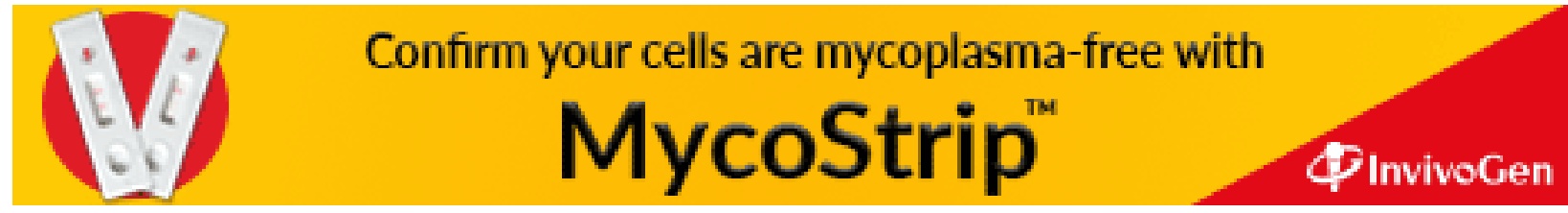

